\title{
Evaluation of Platelet Indices as Early Markers for Diagnosis of Neonatal Sepsis
}

Wafaa Fathy Elsaaed ${ }^{1}$, Asmaa Mohamed Hosny Esh', Abdulfatah Faraj Bin Aeshah ${ }^{1}$, Mohamed Ahmed Arafa ${ }^{1}$

Departments of ${ }^{1}$ Pediatrics and ${ }^{2}$ Clinical Pathology, Faculty of Medicine - Zagazig University, Zagazig, Egypt

*Corresponding author: Abdulfatah Faraj Bin Aeshah, Mobile: (+20)01554929859, E-mail: fattahbenaisha@ gmail.com

\begin{abstract}
Background: Neonatal septicemia is a clinical syndrome characterized by signs and symptoms of infection with or without accompanying bacteremia in the first month of life. Sepsis is a common complication in the neonatal intensive care unit. It is caused by various organisms invading the blood stream, which may be by bacterial, viral, fungal and protozoal infections.
\end{abstract}

Objective: The aim of this study was to diagnose neonatal sepsis early in order to reduce morbidity and mortality of newborn.

Patients and methods: This study was a case-control study that included 132 participants divided into two groups, each group included 66 patients. $1^{\text {st }}$ group included neonatal sepsis and $2^{\text {nd }}$ group was control group without sepsis. They were admitted at Neonatal Unit of Pediatric Department at Zagazig University Hospitals during the period from 11/2019 to 11/2020. Investigations done for all these neonates, and included complete blood count [PLT, PDW, MPV and Platelet-large cell ratio (P-LCR)], CRP, ESR, blood culture, urine culture and chest x-ray.

Results: There was statistically significant difference between the septic cases and control group regarding platelets distribution width (PDW) where 87.9\% of the septic cases had elevated PDW while 45.5\% of control group had elevated PDW. There was statistically significant negative correlation between platelets count with CRP, platelets distribution width, mean platelet volume and P-LCR (increased platelets count is associated with decrease in CRP, platelets distribution width, mean platelet volume and P-LCR) among the control group.

Conclusion: Platelet indices, as part of a routine automated cell count, are cheap and readily available tests that can be used as valuable clues in the diagnosis of neonatal sepsis.

Keywords: Mean platelets volume (MPW), Neonatal sepsis, Platelet distribution width (PDW).

\section{INTRODUCTION}

Neonatal sepsis is an important cause of neonatal morbidity and mortality worldwide. However, it is a diagnostic challenge as there is overlapping signs and symptoms which preclude a specific diagnosis of sepsis. So, we have to rely on investigations to guide us. Blood culture has always been the gold standard for the diagnosis of neonatal sepsis. It has been noted that only $20 \%$ of symptomatic neonates with suspected early-onset sepsis (EOS) have a positive blood culture, and only 30\% neonates clinically suspected to have late-onset sepsis (LOS) in neonatal intensive care unit (NICU) setting have a positive blood culture ${ }^{(\mathbf{1})}$.

According to the age, neonatal sepsis is divided into early-onset sepsis ( $\leq 3$ days of birth) and late onset sepsis (After 3 days). The early-onset sepsis usually results from organisms acquired intrapartum, but late-onset sepsis is usually acquired from the environment ${ }^{(3)}$. Neonatal sepsis occurs in one to four cases per 1000 live births in the developed countries, while it has been 10 times greater in many developing countries ${ }^{(4)}$.

Almost all body systems and organs including haemostatic system can be affected by sepsis. In this situation, clotting cascades do not function. Many inflammatory cytokines are released from endothelial and mononuclear cells.
Thrombosis takes place at later stages and stimulation of plasminogen and activation of antithrombin occurs in fibrinolytic system (5). Moreover, bacteria or its products may cause damage to endothelial leading to platelet adhesion and aggregation or may bind directly to platelets leading to aggregation and expedited elimination from blood circulation ${ }^{(6)}$. Group B Streptococcus, Escherichia coli, coagulase-negative Staphylococcus, Staphylococcus aureus, Klebsiella, Pseudomonas and Enterobacter are the most common cause of neonatal sepsis $^{(7)}$.

Platelet distribution width (PDW) is an indicator of variation in platelet size. Normal ranges of PDW are between $10 \%$ and $17 \%$. The PDW levels increases in platelet consumption when turnover is increased, and behavior similar to MPV during acute severe infections. Platelet-large cell ratio (P-LCR) indicates the proportion of platelets greater than $12 \mathrm{fL}$ and the normal range is below 30 percent in the total platelet count.

These indices can be measured by an inexpensive and readily available routine blood count ${ }^{(8)}$. This study aimed to early diagnose neonatal sepsis to reduce morbidity and mortality of newborn. 


\section{PATIENTS AND METHODS}

This case-control study included 132 participants divided into two groups, each group included 66 patients. $1^{\text {st }}$ group with neonatal sepsis and $2^{\text {nd }}$ group was control group without sepsis. The study was conducted in the Neonatal Unit of Pediatric Department, Zagazig University Hospitals during the period from $11 / 2019$ to $11 / 2020$.

\section{Ethical approval:}

Written informed consent was obtained from all participants' parents and the study was approved by the Research Ethical Committee of Faculty of Medicine, Zagazig University.

The work has been carried out in accordance with The Code of Ethics of the World Medical Association (Declaration of Helsinki) for studies involving humans.

\section{Inclusion criteria:}

Gestational age $\geq 32$ weeks. Post natal age from birth to 28 days. Neonates average weight $\geq 1.5 \mathrm{~kg}$. Any suspected case of neonatal sepsis with maternal risk factor for sepsis e.g., prolonged labour, PROM, maternal intra partum fever, UTI and chorioaminionitis. Neonates with clinical sepsis (apnea, poor sucking, reduced activity, lethargy, hypotonia, hyporeflexia, cyanosis, respiratory distress, irritability, hypothermia, hyperthermia and not doing well neonates). Neonates who suffer from diseases other than suspected sepsis as jaundice, TTN, meconium aspiration etc...and history of chorioamnionitis.

Exclusion criteria: Newborn above 28 days. Absence of informed consent. Neonates who received antibiotics before admission.

All patients were subjected to full history taking, clinical examination (physical examination, vital signs, anthropometric measurement) and laboratory investigations that included complete blood count (CBC). $1 \mathrm{ml}$ blood samples were taken from all of the participants and collected in tubes containing EDTA. The CBC was performed using a Sysmex XT1800i analyser (Germany).

C - reactive protein (CRP): Samples were directly collected into EDTA-coated tubes. Plasma was obtained by centrifugation at $4^{\circ} \mathrm{C}$ for $15 \mathrm{~min}$ at $1000 \mathrm{xg}$, and stored at $-80{ }^{\circ} \mathrm{C}$ until use. Freezing/thawing cycles were carefully avoided.

\section{Blood culture:}

Blood samples were taken at time of presentation of sepsis. Aerobic and anaerobic cultures were done on blood agar plates at $10 \% \mathrm{CO}_{2}$ and on MacConkey agar plates. Isolated colonies were further identified by examination of their colony morphology, gram stained smears, biochemical and enzymatic reactions. True bacteremia was considered when the blood culture was positive within 72 hours.

If no growth was detected, the sample was incubated up to 10 days with further subcultures every other day on solid media. If no growth appeared after 10 days of incubation, blood culture was considered negative. Antibiotic sensitivity test was done by Kirby Baur technique.

Urine culture: Urine sample were collected by supra pubic aspiration. All the samples were sent to the laboratory within half-hour of the procedure.

L.P: if needed; Cerebrospinal fluid examination should be done in all neonates with suspected meningitis and CSF cultures to diagnose bacteria, fungi, and viruses meningitis ${ }^{(9)}$.

\section{Statistical Analysis}

Collected data were recorded then presented and analyzed statistically by computer using SPSS version 22 (SPSS Inc. Chicago, IL, U.S.A).

Data were presented in tables and graphs as median and mean \pm standard deviation for quantitative variables and as number and percentage for qualitative variables. Chi-square test and Fisher's exact test, wherever appropriate, were used for data analysis. Independent two-sample t -tests or MannWhitney-U tests were applied to compare the continuous variables between the two groups. Kruskal Wallis test was used for comparing the median. $\mathrm{P}$ value equal to or less than 0.05 was considered statistically significant.

\section{RESULTS}

Table (1) showed that there was statistically significant difference between the septic cases and control group regarding age and gestational age where sepsis was among neonates with older age $(5.7 \pm 5.1$ versus $2.4 \pm 1.6$ days) and lesser gestational age (36.5 \pm 1.3 versus $37.4 \pm 0.9$ weeks) but regarding sex distribution, there was no statistically significant difference between the septic cases and control group. 
Table (1): Demographic characteristics and gestational age between septic group and control groups

\begin{tabular}{|c|c|c|c|c|c|c|}
\hline Variable & \multicolumn{2}{|c|}{ Case (66) } & \multicolumn{2}{|c|}{ Control (66) } & Test & p-value \\
\hline $\begin{array}{c}\text { Age (days) } \\
\text { Mean } \pm \text { SD } \\
\text { (Range) } \\
\text { Median }\end{array}$ & \multicolumn{2}{|c|}{$\begin{array}{c}5.7 \pm 5.1 \\
(1-20) \\
3\end{array}$} & \multicolumn{2}{|c|}{$\begin{array}{c}2.4 \pm 1.6 \\
(1-10) \\
2\end{array}$} & $\begin{array}{r}\mathrm{M} \cdot \mathrm{W}= \\
4.5\end{array}$ & $0.001 * *$ \\
\hline $\begin{array}{l}\begin{array}{l}\text { Gestational age } \\
\text { (weeks) }\end{array} \\
\text { Mean } \pm \text { SD } \\
\text { (Range) } \\
\text { Median } \\
\end{array}$ & \multicolumn{2}{|c|}{$\begin{array}{c}36.5 \pm 1.3 \\
(33-38) \\
37\end{array}$} & \multicolumn{2}{|c|}{$\begin{array}{c}37.4 \pm 0.9 \\
(35-39) \\
38\end{array}$} & $\mathrm{~T}=4.5$ & $0.001 * *$ \\
\hline \multirow{2}{*}{ Variable } & \multicolumn{2}{|c|}{ Case } & \multicolumn{2}{|c|}{ Control } & \multirow{2}{*}{$\chi^{2}$} & \multirow{2}{*}{ p-value } \\
\hline & No(66) & $\%$ & $\operatorname{No}(66)$ & $\%$ & & \\
\hline $\begin{array}{c}\text { Male (78) } \\
\text { Female (54) }\end{array}$ & $\begin{array}{l}44 \\
22\end{array}$ & $\begin{array}{l}66.7 \% \\
33.3 \%\end{array}$ & $\begin{array}{l}34 \\
32\end{array}$ & $\begin{array}{l}51.5 \% \\
48.5 \%\end{array}$ & 2.5 & 0.1 \\
\hline
\end{tabular}

M.W= Mann-Whitney U test

** Statistically highly significant difference $(\mathrm{P} \leq 0.001)$

Figure (1) showed that streptococcus agalactiae causing pneumonia was the commonest organism (30.3\%) among septic cases followed by klebsilla and staph epidermidis; each of them (24.2\%) then pseudomonas aeruginosa $(9.1 \%)$ and lastly staph hominis and candida $(6.1 \%)$ for each.

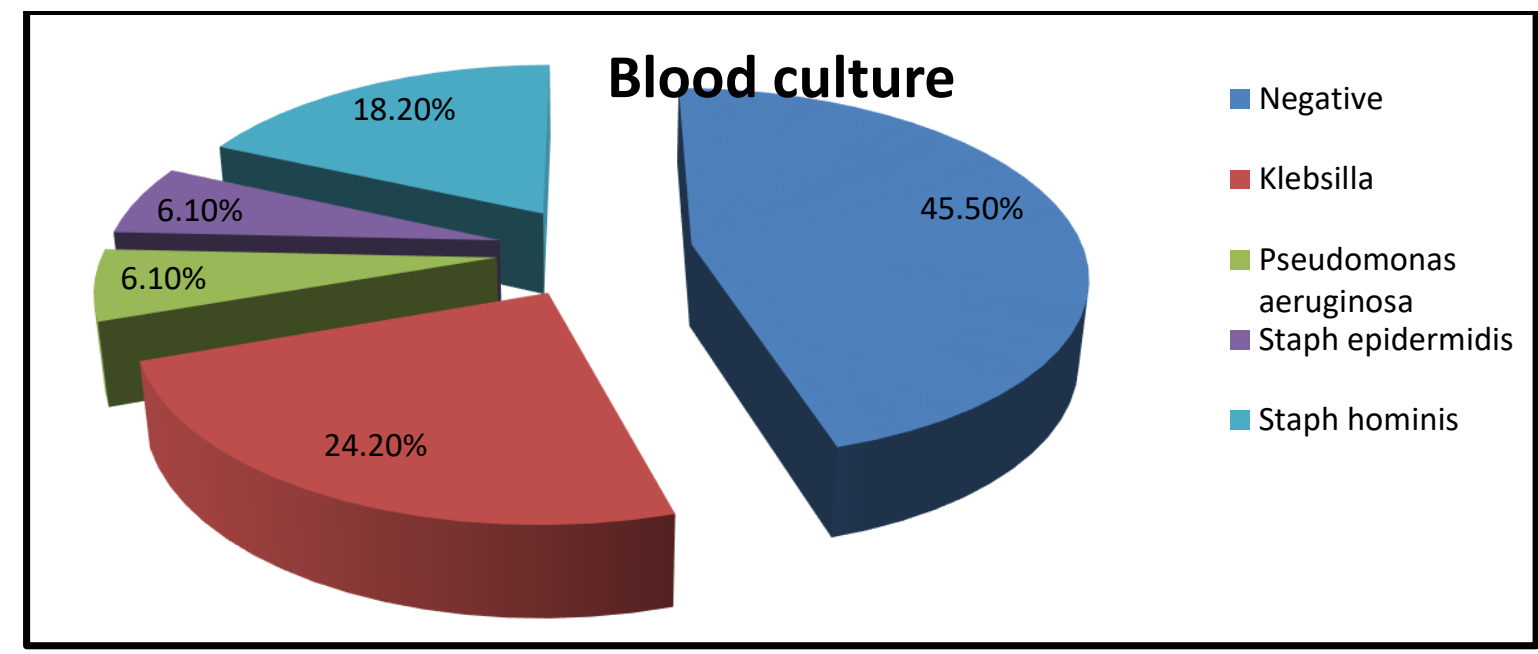

Fig. (1): Pie chart for isolated organisms form blood culture of the septic cases

Table (2) showed that there was statistically significant decrease in platelets count among the septic cases than control group $(281.3 \pm 145.9$ versus $343.9 \pm 134.8)$ but regarding PDW and P-LCR, they were statistically significant increased among the septic cases than control group $(16.6 \pm 5.6$ versus $11.8 \pm 2.1$ and32.3 \pm 4.3 versus $21.7 \pm 5.7$ respectively). In regard to MPV, there was no statistically significant difference between the septic case and control group.

Table (2): Comparing platelets indices between case and control groups

\begin{tabular}{||l|c|c|c|c||}
\hline Variable & Case (66) & Control (66) & Test & p-value \\
\hline $\begin{array}{l}\text { Platelets count }(/ \mu \mathrm{L}) \\
\text { Mean } \pm \text { SD }\end{array}$ & $281.3 \pm 5.9$ & $343.9 \pm 4.8$ & $\mathrm{M} . \mathrm{W}=2.5$ & $\mathbf{0 . 0 1 *}$ \\
\hline $\begin{array}{l}\text { Platelets distribution width (PDW) (\%) } \\
\text { Mean } \pm \text { SD }\end{array}$ & $16.6 \pm 3.1$ & $11.8 \pm 2.1$ & $\mathbf{T}=6.5$ & $\mathbf{0 . 0 0 1 * *}$ \\
\hline $\begin{array}{l}\text { Mean platelet volume } \\
\text { (MPV)(fl) } \\
\text { Mean } \pm \text { SD }\end{array}$ & $10.7 \pm 0.8$ & $10.4 \pm 2$ & $\mathbf{T}=1.1$ & 0.3 \\
\hline $\begin{array}{l}\text { Platelet large cell ratio (P-LCR) (\%) } \\
\text { Mean } \pm \text { SD }\end{array}$ & $32.3 \pm 4.3$ & $21.7 \pm 4.7$ & $\mathbf{T}=11.9$ & $\mathbf{0 . 0 0 1 * *}$ \\
\hline
\end{tabular}

M.W= Mann-Whitney U test

** Statistically highly significant difference $(\mathrm{P} \leq 0.001)$

Table (3) showed that platelets distribution width (PDW), platelets count, mean platelet volume and P-LCR were statistically significantly diagnostic markers for prediction of the septic neonates. 
Table (3): The predictive ability of platelets count, PDW, MPV and P-LCR for the prediction of neonatal sepsis

\begin{tabular}{|c|c|c|c|c|}
\hline Variable & Cut off & AUC & p & 95\% CI \\
\hline Platelets count $(/ \mu \mathrm{L})$ & $<150$ & 0.35 & $\mathbf{0 . 0 0 4} *$ & $(0.26-0.45)$ \\
\hline PDW $(\%)$ & $>11.8$ & 0.83 & $\mathbf{0 . 0 0 1} * *$ & $(0.76-0.89)$ \\
\hline MPV $(\mathbf{f l})$ & $>10.1$ & 0.63 & $\mathbf{0 . 0 1} *$ & $(0.52-0.73)$ \\
\hline P-LCR $(\%)$ & $>25.6$ & 0.94 & $\mathbf{0 . 0 0 1} * *$ & $(0.91-0.98)$ \\
\hline
\end{tabular}

MPV: Mean platelet volume PDW: Platelet Distribution Width

P-LCR: Platelet Large Cell Ratio

Table (4) showed that P-LCR were the most accurate diagnostic marker $(87 \%)$ followed by platelets distribution width (PDW) $(69.9 \%)$ then mean platelet volume $(62.5 \%)$ and lastly platelets count $(48.5 \%)$.

Table (4): The diagnostic value analysis for the ability of platelets count, PDW, MPV and P-LCR for the prediction of neonatal sepsis

\begin{tabular}{|c|c|c|c|c|c|}
\hline Variable & Sensitivity & Specificity & PVP & PVN & Accuracy \\
\hline Platelets count $(/ \mu \mathrm{L})$ & $81.0 \%$ & $16.0 \%$ & $49.1 \%$ & $45.7 \%$ & $48.5 \%$ \\
\hline PDW (\%) & $87.9 \%$ & $52.0 \%$ & $64.7 \%$ & $81.1 \%$ & $69.9 \%$ \\
\hline MPV (fl) & $78.9 \%$ & $46.0 \%$ & $59.4 \%$ & $68.6 \%$ & $62.5 \%$ \\
\hline P-LCR (\%) & $97 \%$ & $77 \%$ & $80.8 \%$ & $96.3 \%$ & $87 \%$ \\
\hline
\end{tabular}

PDW: Mean platelet volume $\quad$ MPV: Mean platelet volume

PVN: predictive value negative PVP: predictive value positive

P-LCR: Platelet large cell ratio

Table (5) showed that $100.0 \%$ of the septic cases had high CRP while only $18.2 \%$ of control group had high CRP. There was statistically significant difference between the septic cases and control group regarding P-LCR where $97.0 \%$ of the septic cases had elevated P-LCR, while only $33.3 \%$ of control group had elevated P-LCR. There was statistically significant difference between the septic cases and control group regarding mean platelet volume where $(78.8 \%)$ of the septic cases had elevated MPV while $(48.5 \%)$ of control group had elevated MPV. There was statistically significant difference between the septic cases and control group regarding platelets distribution width (PDW) where $87.9 \%$ of the septic cases had elevated PDW, while $45.5 \%$ of control group had elevated PDW. There was no statistically significant difference between the septic cases and control group regarding platelets count where $18.2 \%$ and $15.2 \%$ of the septic cases and control group respectively had decreased platelets count.

Table (5): Relation between sepsis and (CRP, P-LCR, MPV, PDW platelets count) according to the cut off value between septic group and control group

\begin{tabular}{|c|c|c|c|c|c|c|c|}
\hline & \multicolumn{2}{|c|}{ Septic group } & \multicolumn{2}{|c|}{ Control group } & \multirow{2}{*}{$\chi^{2}$} & \multirow{2}{*}{ p-value } & \multirow{2}{*}{$\begin{array}{c}\text { Odds } \\
(95 \% \mathrm{CI})\end{array}$} \\
\hline & $\operatorname{No}(66)$ & $\%$ & $\operatorname{No}(66)$ & $\%$ & & & \\
\hline \multicolumn{8}{|l|}{ CRP } \\
\hline$>6(78)$ & 66 & $100.0 \%$ & 12 & $18.2 \%$ & \multirow{2}{*}{$F E T$} & \multirow{2}{*}{$0.001 * *$} & 0.15 \\
\hline$<6(54)$ & 0.0 & $0.0 \%$ & 54 & $81.8 \%$ & & & $(0.09 \%-0.26 \%)$ \\
\hline \multicolumn{8}{|l|}{$P-L C R$} \\
\hline$>25.6 \%(86)$ & 64 & $97.0 \%$ & 22 & $33.3 \%$ & \multirow{2}{*}{$F E T$} & \multirow{2}{*}{$\begin{array}{c}0.001 \\
* *\end{array}$} & \multirow{2}{*}{$\begin{array}{c}64 \\
(14.3 \%-28.6 \%)\end{array}$} \\
\hline$<25.6 \%(46)$ & 2 & $3.0 \%$ & 44 & $66.7 \%$ & & & \\
\hline \multicolumn{8}{|l|}{ MPV } \\
\hline$>10.1(f l)(86)$ & 52 & $78.8 \%$ & 32 & $48.5 \%$ & \multirow{2}{*}{13.9} & \multirow{2}{*}{$0.001 * *$} & 3.9 \\
\hline$<10.1(f l)(46)$ & 14 & $21.2 \%$ & 34 & $51.5 \%$ & & & $(1.8 \%-8.5 \%)$ \\
\hline \multicolumn{8}{|l|}{ PDW } \\
\hline$>11.8 \%(88)$ & 58 & $87.9 \%$ & 30 & $45.5 \%$ & \multirow{2}{*}{26.7} & \multirow{2}{*}{$0.001 * *$} & 8.7 \\
\hline$<11.8 \%$ & 8 & $12.1 \%$ & 36 & $54.5 \%$ & & & $(3.6 \%-21.1 \%)$ \\
\hline \multicolumn{8}{|l|}{ Platelets count } \\
\hline$<150(/ \mu \mathrm{L})(88)$ & 12 & $18.2 \%$ & 10 & $15.2 \%$ & \multirow[b]{2}{*}{0.2} & \multirow{2}{*}{0.6} & \multirow{2}{*}{$\begin{array}{c}1.2 \\
(0.49 \%-3.1 \%)\end{array}$} \\
\hline$>150(/ \mu \mathrm{L})(44)$ & 54 & $\mathbf{8 1 . 8 \%}$ & 56 & $84.8 \%$ & & & \\
\hline
\end{tabular}

**FET $=$ Fisher Exact test $* * M P V:$ Mean platelet volume

$* *$ Statistically highly significant difference $(\mathrm{P} \leq 0.001) * * \chi^{2}$ : chi square test $\quad * * * * \chi^{2}$ : chi square test

This study showed that there was statistically significant negative correlation between platelets count with mean platelet volume and P-LCR (increased platelets count was associated with decrease in mean platelet volume and PLCR) among the septic cases. Regarding other variables, there was no statistically significant correlation with platelets count among the septic cases (Table 6). 
Table (6): Correlation between platelets count with patients' characteristics and laboratory investigations among the septic group

\begin{tabular}{|l|c|c|c|}
\hline \multirow{2}{*}{\multicolumn{1}{|c|}{ Variable }} & \multicolumn{3}{c|}{ Platelets count } \\
\cline { 2 - 4 } & $\mathbf{r}^{\wedge}$ & $\mathbf{p}$ & Significant \\
\hline Age & 0.01 & $>0.05$ & NS \\
\hline $\begin{array}{l}\text { Gestational } \\
\text { age }\end{array}$ & 0.03 & $>0.05$ & NS \\
\hline WBCs $(/ \mu \mathrm{L})$ & 0.01 & $>0.05$ & NS \\
\hline CRP & 0.02 & $>0.05$ & NS \\
\hline PDW (\%) & -0.1 & $>0.05$ & NS \\
\hline MPV (fl) & $\mathbf{- 0 . 3}$ & $\mathbf{0 . 0 1 *}$ & S \\
\hline P-LCR $(\%)$ & $\mathbf{- 0 . 3}$ & $\mathbf{0 . 0 0 4 *}$ & $\mathbf{S}$ \\
\hline
\end{tabular}

** Statistically significant difference $(\mathrm{P} \leq 0.05)$ **SIG: significant

$* * r$ : correlation

\section{DISCUSSION}

The current study showed that there was statistically significant difference between the septic cases and control group regarding age and gestational age, where sepsis was among neonates with older age (5.7 \pm 5.1 versus $2.4 \pm 1.6$ days) and lesser gestational age $(36.5 \pm 1.3$ versus $37.4 \pm 0.9$ weeks $)$. But, regarding sex distribution, there was no statistically significant difference between the septic cases and control group. This is in agreement with Madani $\boldsymbol{e t}$ $\boldsymbol{a l} .^{(\mathbf{1 0})}$ who found that the mean age was significantly higher than control group (7.5 versus 2.6 days, pvalue $=0.04 *$ ) with no significant difference regarding sex with male to female ratio was (10.10 versus 12.8 , p-value $=0.6$ )

The isolated organisms among the septic group were as follow: streptococcus agalactiae causing pneumonia was the commonest organism $(30.3 \%)$ followed by klebsilla and staph epidermidis; each of them $(24.2 \%)$ then pseudomonas aeruginosa $(9.1 \%)$ and lastly staph hominis and Candida $(6.1 \%)$ for each respectively.

In regard to mean platelet volume (MPV), there was no statistically significant difference between the septic cases and control group. This is consistent with Tayman et al. ${ }^{(11)}$ whose results support that platelet count, MPV and PDW were significantly different between sepsis and control groups $(270.8 \pm 98$ versus $240 \pm 59,8.3 \pm 0.3$ versus $10.3 \pm 0.9$ and $16.6 \pm 0.2$ versus $17.3 \pm 0.1$, p-value < $0.05)$ respectively. Finally, Mittal et al. ${ }^{(12)}$ concluded that the cases group had a greater number of subjects with higher platelet indices than the control group $(\mathrm{P}$ $<0.05)$, where the mean platelet count was lower in cases group $\left(1.098 \pm 0.747 \mathrm{lakhs} / \mathrm{mm}^{3}\right)$ than in the control group $\left(2.038 \pm 0.762\right.$ lakhs $\left./ \mathrm{mm}^{3}, \mathrm{P}<0.0001\right)$. The mean MPV was higher in the case group (11.85 $\pm 1.716 \mathrm{fl})$ than in the control group $(9.81 \pm 1.460 \mathrm{fl})$, $\mathrm{P}<0.0001$. In addition, the mean PDW value was higher in the case group $(20.68 \pm 2.239)$ than in the control group $(18.69 \pm 1.974), \mathrm{P}<0.0001$.
Regarding the diagnostic ability of platelet indices, the current study found that P-LCR was the most accurate diagnostic marker $(87 \%)$ followed by PDW (69.9\%) then MPV (62.5\%) and lastly platelets count $(48.5 \%)$. This is similar to Madani et al. ${ }^{(10)}$ who found that P-LCR was the most accurate diagnostic marker followed by PDW then MPV where their sensitivity and specificity were $65.2 \%$ \& $80.0 \%, 70.0 \%$ \& $70.5 \%$ and $65.3 \%$ \& $75.0 \%$ respectively.

Oppositely the current result was in contrast with Mittal et al. ${ }^{(\mathbf{1 2})}$ who assessed the sensitivity and specificity of platelet indices for diagnosis of neonatal sepsis by comparing them against blood culture, which was the gold standard for diagnosis of neonatal sepsis and found that thrombocytopenia was the most sensitive marker (83.08\%) followed by MPV and PDW in detecting babies with culture positive sepsis. However, it had a low specificity (20.33\%). But when we combined MPV and PDW or combined all the three markers (MPV + PDW + PC), the specificity increased to $46.34 \%$. The area under curve (AUC) was 0.156 for platelet count, 0.814 for MPV and 0.743 for PDW on ROC curves.

Concerning elevated platelet indices, the current study found that there was statistically significant difference between the septic group and control group regarding elevated P-LCR, MPV and PDW where $97.0 \%, 78.8 \%$ and $87.9 \%$ of the septic cases had elevated P-LCR while only $33.3 \%$, 48.5\% and $45.5 \%$ of control group had elevated P-LCR. There was no statistically significant difference between the septic cases and control group regarding platelets count where $18.2 \%$ and $15.2 \%$ of the septic cases and control group respectively had decreased platelets count. This is in similarity with Karne et al. (13) who found that out of total sepsis positive cases, severe thrombocytopenia was presented in $57.5 \%$, while mild and moderate thrombocytopenia in $22.5 \%$ and $20.0 \%$ respectively. Non infected neonates had majority of mild thrombocytopenia in $74.5 \%$ and severe thrombocytopenia in $14.9 \%$. Similarly, Arif $\boldsymbol{e t}$ al. ${ }^{(14)}$ showed that thrombocytopenia was seen in $83.5 \%$ of neonates' cases with sepsis.

Our study found that there was statistically significant negative correlation between platelets count with MPV and P-LCR (increased platelets count is associated with decrease in MPV and P-LCR) among the septic cases. Regarding other variables, there was no statistically significant correlation with platelets count among the septic cases. This is in consistence with Tayman et al. ${ }^{(11)}$ whose results found that platelets count was correlated negatively with MPV (Rho $=-0.852, \mathrm{p}=0.01)$ and PDW (Rho $=-0.765, \mathrm{p}=0.014)$, and a positive correlation was found between MPV and PDW (Rho $=0.791, \mathrm{p}=$ $0.022)$. 


\section{CONCLUSION}

Platelet indices, as part of a routine automated cell count, are cheap and readily available tests that can be used as valuable clues in the diagnosis of neonatal sepsis. P-LCR was the most accurate diagnostic marker followed by platelets distribution width (PDW) then mean platelet volume (MPV) and lastly platelets count (48.5\%). Platelets indices did not differ significantly with gestational age nor with Gram-positive or Gram-negative blood cultures.

\section{REFERENCES}

1. Singh S, Dutta S, Narang A et al. (2003): Predictive clinical scores for diagnosis of late onset neonatal septicaemia. J Trop Pediatr., 49: 235-9.

2. Okascharoen C, Sirinavin S, Thakkinstian A et al. (2005): A bedside prediction Scoring model for late onset neonatal sepsis. J Perinatol., 25: 778-83.

3. Chiarot E, Spagnuolo A, Maccari S et al. (2018): Protective effect of Group B Streptococcus type-III polysaccharide conjugates against maternal colonization, ascending infection and neonatal transmission in rodent models. Scientific Reports, 8 (1): 2593-98.

4. Simonsen $\mathrm{K}$, Anderson-Berry A, Delair $\mathrm{S}$ et al. (2014): Early-onset neonatal sepsis. Clinical Microbiology Reviews, 27 (1): 21-47.

5. Zardi E, Zardi D, Dobrina A et al. (2007): Prostacyclin in sepsis: a systematic review. Prostaglandins \& Other Lipid Mediators, 83 (1-2): 124.

6. Stevenson E, Rubenstein A, Radin G et al. (2014): Two decades of mortality trends among patients with severe sepsis: a comparative meta-analysis. Critical Care Medicine, 42 (3): 625-31.

7. Rostami-Far Z, Ghadiri K, Rostami-Far M et al. (2016): Glucose-6-phosphate dehydrogenase deficiency (G6PD) as a risk factor of neonatal sepsis. Journal of Medicine and Life, 9 (1): 34-8.

8. Sachdev R, Tiwari A, Goel S et al. (2014): Establishing biological reference intervals for novel platelet parameters (immature platelet fraction, high immature platelet fraction, platelet distribution width, platelet large cell ratio, platelet-X, plateletcrit and platelet distribution width) and their correlations among each other. Indian Journal of Pathology and Microbiology, 57 (2): 231-5.

9. Pearson J, Fuller G (2012): Lumbar punctures and cerebrospinal fluid analysis. Medicine, 40 (8): 459462.

10. Madani S, Amiri S, Khazaei S et al. (2019): platelet indices as useful indicators of neonatal sepsis. Journal of Evolution of Medical and Dental Sciences, 8 (20): 1612-1618.

11. Tayman C, Tonbul A, Akca H et al. (2014): Mean platelet volume (MPV) may simply predict the severity of sepsis in preterm infants. Clin Lab., 60 (7): 1193-200.

12. Mittal A, Arya S, Charan L et al. (2020): Evaluation of platelet indices as additional diagnostic tool for neonatal sepsis. Astrocyte, 4 (4): 205-209.

13. Karne T, Joshi D, Zile U et al. (2017): Study of platelet count and platelet indices in neonatal sepsis in tertiary care institute. MVP Journal of Medical Science, 4 (1): 55-60.

14. Arif S, Ahmed I, Ali $S$ et al. (2012): Thrombocytopenia and bacterial sepsis in neonates. Indian J Hematol Blood Transfusion, 28 (3): 147-51. 